University of South Carolina

Scholar Commons

8-21-2012

\title{
Incorporation of Quantum Effects for Selected Degrees of Freedom into the Trajectory-Based Dynamics Using Spatial Domains
}

\author{
Sophya V. Garashchuk \\ University of South Carolina - Columbia, garashch@mailbox.sc.edu \\ Mikhail V. Volkov \\ University of South Carolina - Columbia
}

Follow this and additional works at: https://scholarcommons.sc.edu/chem_facpub

Part of the Chemistry Commons

\section{Publication Info}

The Journal of Chemical Physics, Volume 137, Issue 7, 2012, pages 074415-.

(c) The Journal of Chemical Physics 2012, American Institute of Physics.

This Article is brought to you by the Chemistry and Biochemistry, Department of at Scholar Commons. It has been accepted for inclusion in Faculty Publications by an authorized administrator of Scholar Commons. For more information, please contact digres@mailbox.sc.edu. 


\title{
Incorporation of quantum effects for selected degrees of freedom into the trajectory-based dynamics using spatial domains
}

\author{
Sophya Garashchuk and Mikhail V. Volkov \\ Department of Chemistry and Biochemistry, University of South Carolina, Columbia, \\ South Carolina 29208, USA
}

(Received 30 May 2012; accepted 2 August 2012; published online 21 August 2012)

\begin{abstract}
The approach of defining quantum corrections on nuclear dynamics of molecular systems incorporated approximately into selected degrees of freedom, is described. The approach is based on the Madelung-de-Broglie-Bohm formulation of time-dependent quantum mechanics which represents a wavefunction in terms of an ensemble of trajectories. The trajectories follow classical laws of motion except that the quantum potential, dependent on the wavefunction amplitude and its derivatives, is added to the external, classical potential. In this framework the quantum potential, determined approximately for practical reasons, is included only into the "quantum" degrees of freedom describing light particles such as protons, while neglecting with the quantum force for the heavy, nearly classical nuclei. The entire system comprised of light and heavy particles is described by a single wavefunction of full dimensionality. The coordinate space of heavy particles is divided into spatial domains or subspaces. The quantum force acting on the light particles is determined for each domain of similar configurations of the heavy nuclei. This approach effectively introduces parametric dependence of the reduced dimensionality quantum force, on classical degrees of freedom. This strategy improves accuracy of the quantum force and does not restrict interaction between the domains. The concept is illustrated for two-dimensional scattering systems, where the quantum force is required to reproduce vibrational energy of the quantum degree of freedom. () 2012 American Institute of Physics. [http://dx.doi.org/10.1063/1.4746156]
\end{abstract}

\section{INTRODUCTION}

The cost of finding a numerically exact solution to the Schrödinger equation for coupled anharmonic systems scales exponentially with the system size which makes this task generally intractable. ${ }^{1,2}$ However, for heavy particles such as nuclei, classical dynamics whose cost scales linearly with the systems size is often accurate and insightful. ${ }^{3}$ Classical dynamics methods have been used to study systems of thousands of atoms, ${ }^{4}$ whereas the largest reactive scattering study has been performed for a system of just five atoms-collision of hydrogen and methane. ${ }^{5,6} \mathrm{We}$ are interested in quantum effects on dynamics for intermediate size systems, where classical dynamics describes general behavior, but the quantummechanical (QM) effects such as tunneling and zero-pointenergy (ZPE) are important for a few light particles, e.g., protons. In this regime, semiclassical and quasiclassical trajectory approaches ${ }^{7,8}$ — based on classical trajectory evolution and incorporating main QM effects-are particularly attractive for several reasons. One reason is that the trajectory description of heavy particles is efficient while the basis representation of highly oscillatory (in the classical limit of $\hbar \rightarrow 0$ ) wavefunctions is not. Another reason is that the initial conditions for the trajectories can be chosen randomly, alleviating the problem of exponential scaling typical of the conventional direct-product basis of quantum dynamics. ${ }^{9}$ Finally, the Newton's equations of motion are easy to solve, especially in Cartesian space.

A conceptually appealing reformulation of the timedependent Schrödinger equation (TDSE) in terms of quan- tum trajectory dynamics due to Madelung, de Broglie, and Bohm, ${ }^{10-12}$ incorporates all QM effects exactly. While generally impractical as a numerical approach to solving the Schrödinger equation exactly this quantum trajectory formulation can provide a universal framework for an approximate treatment of light, quantum, and heavy, nearly classical particles in large molecular systems. The equal-footing description of all degrees of freedom (DoFs) in principle resolves problems of quantum/classical separation among DoFs (Ref. 13) associated with mixed quantum-classical approaches. ${ }^{14-16}$ The quantum trajectory formulation served as a foundation for two mixed quantum-classical Bohmian dynamics approaches: ${ }^{13,17}$ both methods are based on quantum evolution of multiple, reduced dimensionality wavefunctions (defined in the quantum DoFs) guided by the Bohmian trajectories in the full-dimensional space, with quantum corrections for the heavy particles neglected. A conceptually related approach from our group involves multiple quantum trajectory ensembles in the quantum DoFs guided by the trajectories in the classical DoFs. The approach, referred to as energy-conserving mixed quantum/classical (EMQC) trajectory dynamics, conserves energy and recovers the fulldimensional wavefunction. ${ }^{18}$ Multiple ensembles in quantum DoFs are defined by the full-dimensional initial wavefunction and evolve independently of each other-an appealing feature, whose downside is lack of energy exchange or wavefunction transfer between the ensembles. The energy exchange between all DoFs, zero-point energy of vibrational modes and internal energy flow are, of course, essential for 
understanding of many molecular processes, e.g., the bond breaking. ${ }^{19-21}$

In this paper, we implement the quantum trajectory formulation approximately and include the quantum corrections on dynamics into selected DoFs within a single fulldimensional trajectory ensemble. The quantum corrections are defined just for the light particles, yet there is no global averaging over the heavy particles, due to introduction of spatial domains or subspaces dependent on positions of heavy particles. This strategy described in Sec. II, allows cheap lowdimensional estimates of the quantum correction to dynamics while allowing interactions within the wavefunction describing various "channels" of the classical DoFs. This formalism is presented for two Cartesian dimensions: the quantum DoF, labeled $x$, describes a light particle of mass $m$ and the nearly classical DoF, labeled $y$, describes a heavy particle of mass $M$, $m \ll M$. Examples for two-dimensional models of molecular collisions are given in Sec. III. Section IV concludes.

\section{THE QUANTUM TRAJECTORY FORMALISM ON SPATIAL DOMAINS}

The quantum trajectory (also referred to as Bohmian or hydrodynamic) dynamics ${ }^{10-12}$ follows from the TDSE applied to a wavefunction in the polar form,

$$
\psi(x, y, t)=A(x, y, t) \exp (\imath S(x, y, t) / \hbar) .
$$

The wavefunction amplitude, $A$, and phase, $S$, are assumed to be real functions at all times. Substitution of Eq. (1) into TDSE,

$$
\iota \hbar \frac{\partial}{\partial t} \psi(x, y, t)=\hat{H} \psi(x, y, t),
$$

with the Hamiltonian

$$
\hat{H}=\hat{K}+V(x, y), \quad \hat{K}=-\frac{\hbar^{2}}{2 m} \frac{\partial^{2}}{\partial x^{2}}-\frac{\hbar^{2}}{2 M} \frac{\partial^{2}}{\partial y^{2}}
$$

and assignment of the trajectory momentum as the gradient of the wavefunction phase,

$$
p_{x}=\frac{\partial S}{\partial x}, \quad p_{y}=\frac{\partial S}{\partial y}
$$

lead to the Newton's equations of motion for the quantum trajectories:

$$
\begin{array}{ll}
\frac{d x}{d t}=\frac{p_{x}}{m}, & \frac{d p_{x}}{d t}=-\frac{\partial}{\partial x}(V+U), \\
\frac{d y}{d t}=\frac{p_{y}}{M}, & \frac{d p_{y}}{d t}=-\frac{\partial}{\partial y}(V+U) .
\end{array}
$$

The crucial difference with the classical equations of motion, responsible for all quantum effects, is addition of the quantum potential $U$,

$$
U=-\frac{\hbar^{2}}{2 A}\left(\frac{1}{m} \frac{\partial^{2} A}{\partial x^{2}}+\frac{1}{M} \frac{\partial^{2} A}{\partial y^{2}}\right)
$$

to the classical potential $V$. $U$ represents the energy of the wavefunction due to the shape of the wavefunction amplitude. Note that formally $U$ vanishes as particle mass goes to infinity or $\hbar \rightarrow 0$, if the wavefunction amplitude and its derivatives are smooth. Therefore in the limit of heavy particles, it can be viewed as a correction to classical motion. Atomic units of $\hbar, \hbar=1$, are used henceforth. Equations (5) and (6) follow from Eq. (8), which together with the continuity equation of the probability density (9) are equivalent to TDSE (see, for example, Ref. 12 for derivation).

The wavefunction is determined at the trajectory positions by solving equations on its amplitude and phase,

$$
\begin{gathered}
\frac{d S}{d t}=\frac{p_{x}^{2}}{2 m}+\frac{p_{y}^{2}}{2 M}-V-U, \\
\frac{d A^{2}}{d t}=-\left(\frac{1}{m} \frac{\partial p_{x}}{\partial x}+\frac{1}{M} \frac{\partial p_{y}}{\partial y}\right) A^{2} .
\end{gathered}
$$

The full time-derivative in Eqs. (5), (6), (8), and (9) defines the Lagrangian frame of reference,

$$
\frac{d}{d t}=\frac{\partial}{\partial t}+\frac{p_{x}}{m} \frac{\partial}{\partial x}+\frac{p_{y}}{M} \frac{\partial}{\partial y}
$$

Equation (9) describes the continuity of the wavefunction density $A^{2}$, from which it follows that the probability of finding a particle within the volume element associated with a given trajectory $k$ is constant in time. ${ }^{22}$ We refer to this probability as the trajectory weight $w^{k}$,

$$
w^{k}=A^{2}\left(x_{t}^{k}, y_{t}^{k}\right) \delta x_{t}^{k} \delta y_{t}^{k}, \quad \frac{d w_{k}}{d t}=0 .
$$

The trajectory weights are convenient to compute the expectation values, bypassing Eq. (9), once a wavefunction is discretized in terms of the quantum trajectories,

$$
\langle\hat{O}\rangle=\iint\left|\psi\left(x_{t}, y_{t}\right)\right|^{2} O\left(x_{t}, y_{t}\right) d x_{t} d y_{t}=\sum_{k} O\left(x_{t}^{k}, y_{t}^{k}\right) w^{k} \text {. }
$$

Equations (5)-(10) are equivalent to the original TDSE (2) but, generally, impractical for numerical work due to singularities in Eq. (7) when $A(x, y, t)=0$. Thus a cheap approximation which is insensitive (even if inaccurate) to the singularities, yielding linear quantum force (LQF) has been developed. $^{23}$

The LQF is obtained from a global fitting of the nonclassical momentum components,

$$
r_{x}=\frac{1}{A} \frac{\partial A}{\partial x}, r_{y}=\frac{1}{A} \frac{\partial A}{\partial y},
$$

in terms of linear functions from the first and second moments of the trajectory distribution at each time step. Linear approximation to $r_{x}$ and $r_{y}$ is exact for Gaussian wavepackets, allows for wavepacket bifurcations, gives estimates of moderate tunneling and describes ZPE depending on anharmonicity. ${ }^{24,25}$

One way to improve accuracy of the linear approximation described in Ref. 26, is to perform the linear approximation on spatial domains rather than globally on the entire space. To have the Hermitian properties of the kinetic energy operator $\hat{K}$ on a domain defined by the function $\Omega_{l}$, it is explicitly included into the kinetic energy operator $\hat{K}_{l}$. For $\hat{K}$ of Eq. (3) one has

$$
\hat{K}_{l}=\hat{K}-\frac{1}{m \Omega_{l}} \frac{\partial \Omega_{l}}{\partial x} \frac{\partial}{\partial x}-\frac{1}{M \Omega_{l}} \frac{\partial \Omega_{l}}{\partial y} \frac{\partial}{\partial y} .
$$


$\hat{K}_{l}$ acts only on wavefunctions (to the left or to the right of the operator in the inner product) and $\Omega_{l}$ effectively acts as a metric. The domain functions can overlap, but for the rigorous energy conserving formulations must add up to unity,

$$
\sum_{l=1}^{L} \Omega_{l} \equiv 1 .
$$

Equation (14) is used in the variational definition of the approximate quantum potential,

$$
\begin{aligned}
U= & \sum_{l} U_{l} \Omega_{l}, \quad U_{l}=-\frac{1}{2 m}\left(\left(\tilde{r}_{x}^{l}\right)^{2}+\frac{\partial \tilde{r}_{x}^{l}}{\partial x}+\frac{\tilde{r}_{x}^{l}}{\Omega_{l}} \frac{\partial \Omega_{l}}{\partial x}\right) \\
& -\frac{1}{2 M}\left(\left(\tilde{r}_{y}^{l}\right)^{2}+\frac{\partial \tilde{r}_{y}^{l}}{\partial y}+\frac{\tilde{r}_{y}^{l}}{\Omega_{l}} \frac{\partial \Omega_{l}}{\partial y}\right) .
\end{aligned}
$$

Note that the wavefunction is defined via a single quantum trajectory ensemble; the domains are used only in the definition of the quantum potential given by Eq. (16).

Function $\tilde{r}_{x}^{l}$ denotes an approximation to $r_{x}, \tilde{r}_{x}^{l}=\vec{f} \cdot \vec{c}_{x}^{l}$; $\tilde{r}_{y}^{l}$ is defined by analogy to the $x$-component. In the basis of fitting functions $\vec{f}=(1, x, y)$, the vector of fitting coefficients $\vec{c}_{x}^{l}$ for each domain $\Omega_{l}$ is determined from the least squares fit. ${ }^{27}$ The coefficients minimizing the deviation $I=\left\langle\left(r_{x}^{l}-\tilde{r}_{x}^{l}\right)^{2} \Omega_{l}\right\rangle$ are

$$
\left\langle\vec{f} \otimes \vec{f} \Omega_{l}\right\rangle \vec{c}_{x}^{l}=\vec{b}_{x}^{l} .
$$

The components of the RHS vector $\vec{b}_{x}^{l}=\left(b_{1}^{l}, b_{2}^{l}, b_{3}^{l}\right)$ are

$$
\begin{gathered}
b_{x, 1}^{l}=-\frac{1}{2}\left\langle\frac{\partial \Omega_{l}}{\partial x}\right\rangle, \\
b_{x, 2}^{l}=-\frac{1}{2}\left\langle x \frac{\partial \Omega_{l}}{\partial x}\right\rangle-\frac{1}{2}\left\langle\Omega_{l}\right\rangle, \\
b_{x, 3}^{l}=-\frac{1}{2}\left\langle y \frac{\partial \Omega_{l}}{\partial x}\right\rangle .
\end{gathered}
$$

In the context of dynamics of particles with mass separation (quantum and classical DoFs) we can use the idea of spatial domains in a physically meaningful and also simpler way: the domains will be defined in the classical DoFs, so the LQF is determined for the quantum DoFs only from the trajectories belonging to the same classical domain. Thus, the domain functions depend only on the classical DoF, $\Omega_{l}$ $\equiv \Omega_{l}(y)$, and the fitting basis in quantum DoF consists of just two functions, $\vec{f}=(1, x)$. Neglecting with the quantum potential in the classical DoF, the quantum potential of Eq. (16) becomes the following:

$$
U \approx \sum_{l} U_{l}(x) \Omega_{l}(y), \quad U_{l}=-\frac{1}{2 m}\left(\left(\tilde{r}_{x}^{l}\right)^{2}+\frac{\partial \tilde{r}_{x}^{l}}{\partial x}\right)
$$

where approximate functions are determined by Eqs. (17)(19). Note that, formally, the quantum potential of Eq. (21) produces a force on the classical DoF through the derivative of $\Omega_{l}(y)$, if $\tilde{r}_{x}^{l}$ are domain-dependent, which will be the case in approximate dynamics. We will examine the significance of this force in Sec. III. For a two-dimensional system, the simplest choice of domains in the classical DoF is to define strips (or cells for general multidimensional case) in the $y$-coordinate. Labeling the boundaries of $L$ domains with $Y_{l}$, $l=0 \ldots L$, all trajectories-their coordinates are $\left(x_{k}, y_{k}\right)$-for which $Y_{l-1}<y_{k}<Y_{l}$ belong to the $l$ th domain. The quantum potential and force, dependent only on $x$, are computed independently for each domain: one can say that the quantum correction depends on $y$ parametrically through the domain index. The domains are defined by the Heaviside functions,

$$
\Omega_{l}(y)=\theta\left(y-Y_{l-1}\right)-\theta\left(y-Y_{l}\right), \quad Y_{l}>Y_{l-1} .
$$

For numerical stability, the quantum force acting on a trajectory, crossing the domain boundary has to be continuous. Therefore, instead of the Heaviside function we use its smooth version, the Fermi or logistic function as detailed in the Appendix.

\section{COLLISION MODELS AND STABILIZATION OF DYNAMICS}

\section{A. Reactive scattering model}

Long-time description of the zero-point energy, and more generally of the internal vibrational energy flow, is a great challenge for the trajectory-based method. Thus, to illustrate the LQF on domains approach, we start with a simple but relevant to this issue model consisting of a vibrational DoF (as the quantum DoF) whose force constant changes along a reaction coordinate treated as the classical DoF. The vibrational DoF is described by the harmonic oscillator, and there is a barrier along the reaction coordinate:

$$
\begin{gathered}
V=\frac{k(y) x^{2}}{2}+\frac{V_{0}}{\cosh ^{2}(\gamma y)}, \\
k(y)=\frac{k_{2}+k_{1}}{2}+\frac{k_{2}-k_{1}}{2} \tanh (\beta y) .
\end{gathered}
$$

The potential is shown in Fig. 1 and the parameters of $V$ and the initial wavefunction are given in Table I. The reactant region is described by $y<0$. The product channel, $y>0$, is

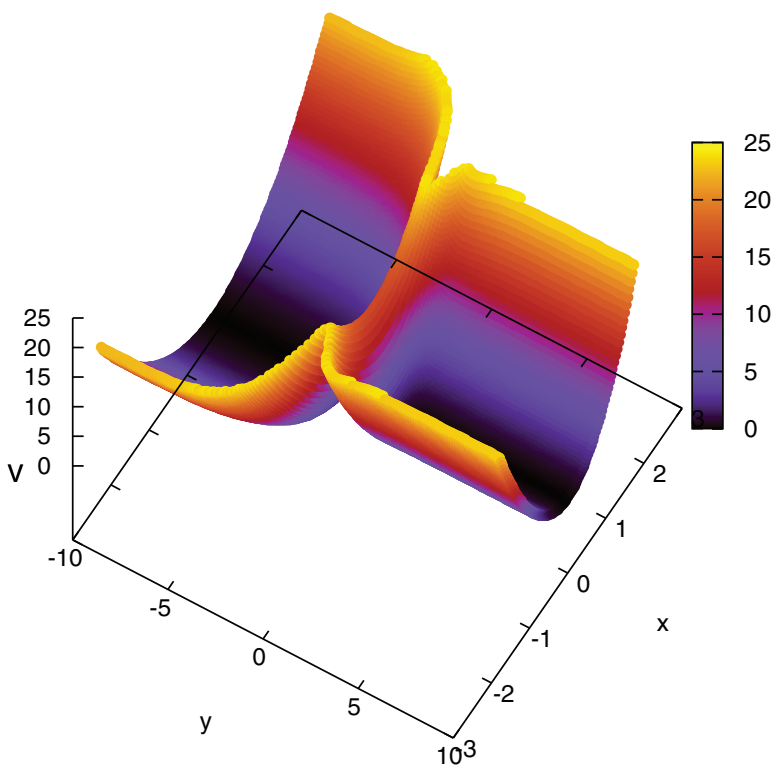

FIG. 1. The scattering potential of Eq. (23). Reactant/product region corresponds to negative/positive $y$-coordinate. 
TABLE I. The potential and wavefunction parameters for the reactive and non-reactive collision models. Particle masses are given in $m_{e}$, the time-step in trajectory calculations dt is given in atomic units. The units of $P_{y}$ are $\sqrt{E_{h} m_{e}}$.

\begin{tabular}{|c|c|c|c|c|c|c|}
\hline \multicolumn{7}{|c|}{$\begin{array}{l}\text { The reactive collision model, Eq. (23) } \\
\text { Parameters of the potential and particle masses }\end{array}$} \\
\hline$k_{1}=5 \frac{E_{h}}{a_{0}^{2}}$ & $k_{2}=15 \frac{E_{h}}{a_{0}^{2}}$ & $\beta=0.5 a_{0}^{-1}$ & $V_{0}=16 E_{h}$ & $\gamma=1.3624 a_{0}^{-1}$ & $m=1$ & $M=10$ \\
\hline \multicolumn{7}{|c|}{ The initial wavefunction, Eq. (24) } \\
\hline$\alpha_{x}=1.118 a_{0}^{-2}$ & $x_{0}=0$ & $P_{x}=0$ & $\alpha_{y}=11.18 a_{0}^{-2}$ & $y_{0}=-3 a_{0}$ & $P_{y}=22$ & $d t=0.025$ \\
\hline \multicolumn{7}{|c|}{ The non-reactive collision model, Eq. (26) } \\
\hline \multicolumn{7}{|c|}{ Parameters of the potential and particle masses } \\
\hline$k=0.264 \frac{E_{h}}{a_{0}^{2}}$ & $x_{e}=2.647 \mathrm{a}_{0}$ & $D=4.34 E_{h}$ & $\gamma=7.82 a_{0}^{-1}$ & $\xi=0.988$ & $m=1813.5$ & $M=6999.0$ \\
\hline \multicolumn{7}{|c|}{ Parameters of the initial wavefunction, Eq. (24) } \\
\hline$\alpha_{x}=10.935 a_{0}^{-2}$ & $x_{0}=x_{e q}$ & $P_{x}=0$ & $\alpha_{y}=1.118 a_{0}^{-2}$ & $y_{0}=-6 a_{0}$ & $P_{y}=[12,32]$ & $d t=0.4$ \\
\hline
\end{tabular}

characterized by a threefold increase in the vibrational force constant. The initial wavefunction is a two-dimensional Gaussian with non-zero momentum in the direction of the barrier,

$$
\begin{aligned}
\psi(x, y, 0)= & \sqrt[4]{\frac{4 \alpha_{x} \alpha_{y}}{\pi^{2}}} \exp \left(-\alpha_{x}\left(x-x_{0}\right)^{2}-\alpha_{y}\left(y-y_{0}\right)^{2}\right. \\
& \left.+\imath P_{x}\left(x-x_{0}\right)+\imath P_{y}\left(y-y_{0}\right)\right) .
\end{aligned}
$$

The considered collision energy yields reaction probability for the wavepacket close to $85 \%$, so we are close to the classical regime in the reactive coordinate. The domains are defined by Eq. (22). The inner step-like domains are taken of equal width, while the outer domains are half-open intervals. The range of domain boundaries is $Y_{l}=[-4,6] \mathrm{a}_{0}$ for $l=1 \ldots L$ $-1 ; Y_{0}=-\infty$ and $Y_{L}=\infty$.

The model of Eq. (23) a quadratic potential in the vibrational DoF and a linear reaction path was chosen to separate the accuracy of the reduced-dimensionality quantum force, dependent on the classical DoF via domains, from the accuracy of the approximate quantum potential in the quantum DoF. The LQF method is exact for Gaussian wavefunctions in a harmonic potential. Anharmonic vibrational potentials could be treated by adding stabilization terms to the LQF dynamics as described in Ref. 28.

Figure 2 shows time-dependent overlap of wavefunction density with that of the asymptotic ground and first excited vibrational states of products (a) and reactants (b) for $L=1$, 2,10 , and 20. Functions $C_{0}(t)$ and $C_{1}(t)$, measuring overlap with the ground and excited vibrational states, respectively, are computed over the trajectory ensemble using Eq. (12),

$$
\begin{aligned}
C_{0}(t) & =\left\langle|\psi|^{2}\left|\chi_{0}\right|^{2}\right\rangle=\sqrt{\frac{2 \alpha}{\pi}} \sum_{k} w^{k} \exp \left(-\alpha\left(x_{t}^{k}-x_{e}\right)^{2}\right), \\
C_{1}(t) & =\left\langle|\psi|^{2}\left|\chi_{1}\right|^{2}\right\rangle \\
& =\sqrt{\frac{2 \alpha}{\pi}} 4 \alpha \sum_{k} w^{k}\left(x_{t}^{k}-x_{e}\right)^{2} \exp \left(-\alpha\left(x_{t}^{k}-x_{e}\right)^{2}\right),
\end{aligned}
$$

where $\alpha=\sqrt{m k_{1}} / 2$ for the projection onto reactants and $\alpha=\sqrt{m k_{2}} / 2$ for the products. $\chi_{0}$ and $\chi_{1}$, which are functions of $x$ centered in this model at $x_{e}=0$, define the ground and excited vibrational eigenstates of the appropriate reaction channel. $C_{0,1}$ are different from the proper projections, defined as $P_{0,1}=\left|\left\langle\psi \mid \chi_{0,1}\right\rangle\right|^{2}$, for which we would need to approximately reconstruct $|\psi(x, y, t)|$ from the trajectories. Direct calculation of projections and wavefunction overlaps, based on the second order local expansion of the wavefunction for which the trajectory position, momentum, action, and weight are known, is described in Ref. 29. We consider the "pseudo"-projections of Eqs. (25) to be able to focus solely on approximations to the dynamics, i.e., to have LQF as the only approximation.

As seen from Fig. 2 the overlaps $C_{0,1}(t)$ converge to exact QM results, obtained with the split-operator/fast fourier transform technique, ${ }^{30,31}$ with respect to the domain width. The convergence with respect to $L$ is not systematic, because we consider variational approximations as part of generally nonlinear dynamics. Formally, the QM limit for the quantum DoF is achieved as the number of trajectories goes to infinity and the domain width goes to zero. For the full QM limit we would also have to include accurate quantum force in the $y$ coordinate. In the current "quasi-classical" treatment of the
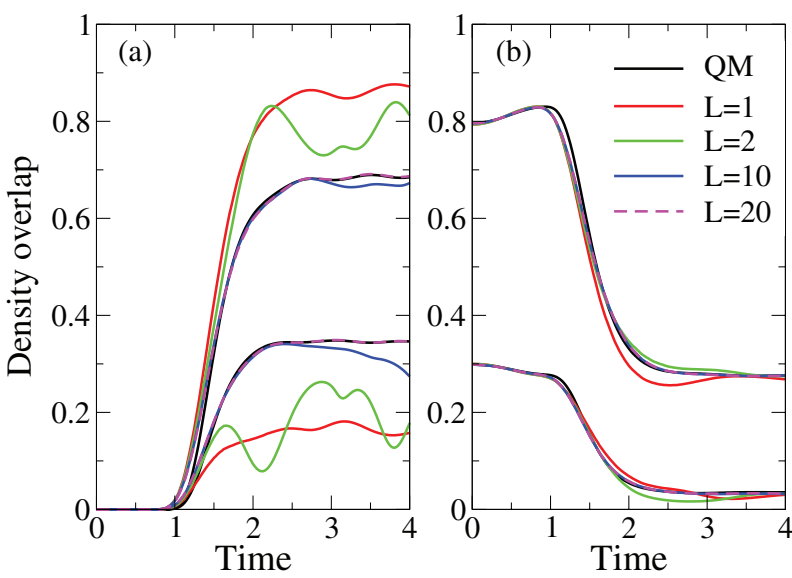

FIG. 2. The density overlaps with the ground and excited vibrational states for the products (a) and reactants (b) obtained from dynamics with $L=1,2$, 10 , and 20 domains, respectively, shown with red, green, blue, and magenta curves. The higher (lower) sets of curves show the overlap with the ground (first excited) state wavefunction density. The ground state overlaps on (b) are shifted by 0.2 in $y$-coordinate for clarity. On both panels exact QM results are shown in black. 
heavy particle we redefine the quantum trajectory momentum in $y$-coordinate to be $p_{y}=P_{y}+2 \alpha_{y}\left(y-y_{0}\right)$, instead of Eq. (4). This modification described in Ref. 18 reproduces exact initial energy of $\psi(x, y, 0)$. Good agreement of $C(t)$ with QM results for $L=10$ and $L=20$ implies that in both reaction channels the ZPE is accurately reproduced. For small number of domains, the ZPE is incorrect but nevertheless non-zero, because for this simple model the trajectories remain coherent in the quantum DoFs.

One shortcoming of the step-like domains is large number of trajectories required for convergence. The results of Fig. 2 were obtained with up to 50000 trajectories (for $L=10$ and 20), which is approximately the number of trajectories required to converge the LQF calculation multiplied by the number of domains. The large number of trajectories was needed, first, to have the domains sufficiently populated for convergence of the variational LQF procedure. Second, we needed about 10 domains to achieve stable trajectory dynamics because the LQF parameters are generally discontinuous at the domain boundaries.

The numerical cost of the LQF-on-domains is essentially linear with respect to the number of trajectories. Typically, the cost of approximate trajectory calculations is comparable to that of exact quantum dynamics. In this application calculation using 5000 trajectories without domains was 3 times faster compared to QM propagation using the split operator on a grid of $128 \times 256$ points. The cost of dynamics with $L$ $=10$ domains took $25 \%$ more time than dynamics on a single domain. However, it required 10 times more trajectories and, thus, was 5 times longer than the exact QM dynamics. The cost of approximate trajectory dynamics is expected to be significantly cheaper than exact QM in higher dimensions.

Below we describe a more challenging application and use continuous domain functions to improve efficiency and stability of the approach.

\section{B. Nonreactive collision model}

The second model describes a non-reactive collision of $\mathrm{He}$ with $\mathrm{HBr}$ in a collinear configuration with the center-ofmass at rest. The stretch of $\mathrm{HBr}$ bond is the quantum DoF and the separation of He and center of mass of the diatomic is the classical DoF. The parameters (listed in Table I) come from the fit of electronic structure data to simple functions,

$$
V=\frac{k\left(x-x_{e}\right)^{2}}{2}+D \exp \left(-\gamma\left(y-\xi\left(x-x_{e}\right)\right)\right) .
$$

The initial wavefunction, given by Eq. (24), is a product of the ground vibrational state with broad wavepacket in the collision coordinate $y$. The wavepacket parameters are listed in Table I. The ratio of effective masses is $M / m=3.86$. We will focus on changes in the vibrational mode induced by the collision with He.

\section{Energy conservation and exchange between the DoFs}

At first, we apply the EMQC approach of Ref. 18 using 50 ensembles of 500 quantum trajectories evolving in $x$

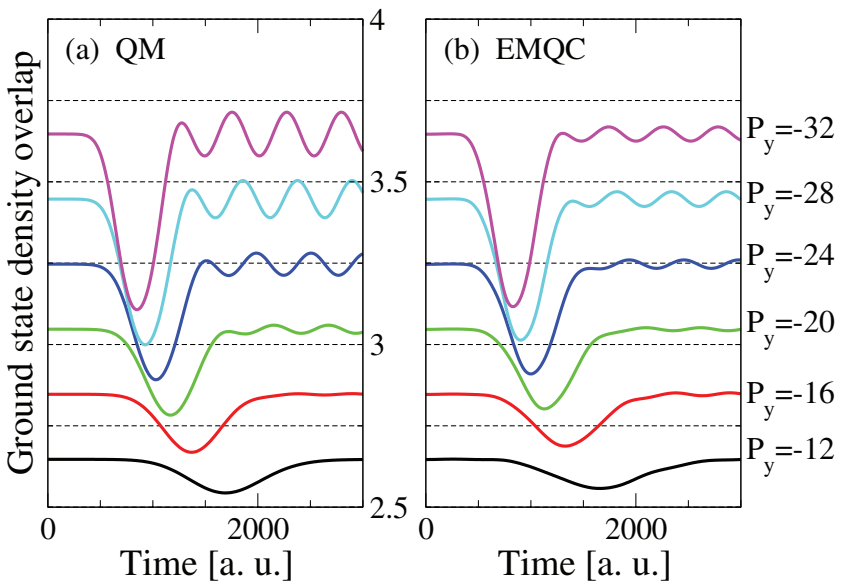

FIG. 3. The average position of the quantum DoF obtained with the exact QM method (a) and using EMQC dynamics (b). The curves correspond to different collision energies defined by $P_{y}=\{-12,-16,-20,-24,-28$, $-32\} \sqrt{E_{h} m_{e}}$ shown in black, red, green, blue, cyan, and magenta, respectively. The curves for consecutive $P_{y}$ are shifted by 0.2 along the vertical axis.

guided by the classical Ehrenfest-like trajectory in $y$. The average positions of the quantum DoF as functions of time are shown in Fig. 3 for several values of collision energies, given by $P_{y}=\{-12,-16,-20,-24,-28,-32\} \sqrt{E_{h} m_{e}}$. The results for consecutive values of $P_{y}$ are shifted along the vertical axis by $0.2 a_{0}$ to avoid overlapping curves. Compared to the split-operator QM calculation displayed on panel (a), the EMQC results on panel (b) show fewer oscillations of $\langle x\rangle$ in time after the collision, while showing good overall agreement. The post-collision $\langle x\rangle$ becomes more oscillatory at larger $\left|P_{y}\right|$, which is explained by the fact that in a fast collision the vibrational wavefunction displaced from equilibrium by the atom-diatom repulsion has less time to return to the ground vibrational state. This behavior is manifested more clearly on Fig. 4 showing the overlaps with the ground vibrational state defined by Eq. (25) with $\alpha=\sqrt{\mathrm{km}} / 2$. The
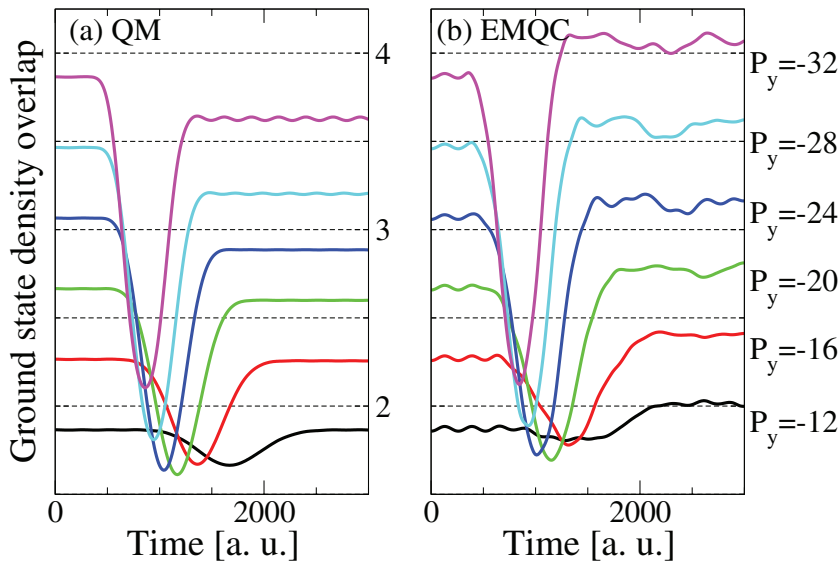

FIG. 4. Density overlap with the ground state of the quantum DoF, obtained with the exact QM method (a) and using EMQC dynamics (b). The curves correspond to different collision energies defined by $P_{y}=\{-12,-16,-20$, $-24,-28,-32\} \sqrt{E_{h} m_{e}}$ shown in black, red, green, blue, cyan, and magenta, respectively. The curves for consecutive $P_{y}$ are shifted by 0.4 along the vertical axis. 
TABLE II. Average quantities for $\mathrm{HBr}+\mathrm{He}$ for $P_{y}=-16 \sqrt{E_{h} m_{e}}$ obtained with various trajectory methods. The results for $\langle x\rangle_{t}$ and deviation $\langle\Delta x\rangle_{t}$ are averaged over the time interval $t=[2250,3000] .\langle y\rangle_{T}$ is given at the final time $T=3000$ a.u. $\Delta E=T^{-1} \int_{0}^{T}|E(t)-E(0)| d t$ for each trajectory method.

\begin{tabular}{lcccccc}
\hline \hline Method & $\mathrm{QM}$ & $\mathrm{LQF}^{2 \mathrm{D}}$ & $\mathrm{LQF}^{1 \mathrm{D}}$ & $\mathrm{LQF}^{d o m}$ & $\mathrm{LQF}^{d e r}$ & $\mathrm{LQF}^{s t b}$ \\
\hline$\langle x\rangle_{t} a_{0}$ & 2.64713 & 2.64417 & 2.64775 & 2.64446 & 2.64578 & 2.64506 \\
$\Delta x_{t} a_{0}$ & 0.00133941 & 0.00813442 & 0.00702329 & 0.00402274 & 0.00761765 & 0.000934789 \\
$\langle y\rangle_{T} a_{0}$ & 6.5538 & 6.2787 & 6.2536 & 6.3752 & 6.1564 & 6.6473 \\
$\Delta E\left[\mathrm{E}_{h}\right]$ & & $1.27 \times 10^{-9}$ & $0.85 \times 10^{-9}$ & $2.91 \times 10^{-4}$ & $1.15 \times 10^{-5}$ & $1.12 \times 10^{-5}$ \\
\hline \hline
\end{tabular}

results for consecutive values of $P_{y}$ are shifted along the vertical axis by 0.4 units. The exact post-collision overlaps, $C_{0}(t)$, are progressively lower than the corresponding $C_{0}(0)$ (left panel). However, in EMQC overlaps the trend is reversedthe overlaps become higher after the collision. We attribute this deficiency, which motivated the use of domains within a single full-dimensional ensemble, to the absence of interaction between the trajectory ensembles corresponding to different classical positions: the energy is conserved for each individual reduced-dimensionality ensemble.

Now we apply the approach of LQF on domains of Sec. II using continuous domain functions given by Eqs. (A2)-(A4). The domain parameters are chosen for overall agreement of the average positions and overlaps of the wavefunction density with the vibrational ground state compared to the QM results for $P_{y}=-16 \sqrt{E_{h} m_{e}}$. We have examined dependence of the overlap with the ground vibrational state density, $C_{0}(t)$, for $\beta=\{5,10,15,20\} a_{0}^{-1}$ and for the number of domains $L=\{5,10,15,20\}$ whose boundaries were equally distributed in the potential coupling region between $2<y<10 a_{0}$. For fixed $L$ the deviations from the average value after the collision due to $\beta$ were $3 \%, 2 \%, 4 \%$, $4 \%$ for the respective $L$. Deviations from the average due to $L$ for fixed $\beta$ values were between $0.5 \%$ and $2.5 \%$. Larger values of $\beta$ and $L$ had poor convergence and oscillatory behavior in $\langle x\rangle$ after the collision. Thus we have chosen $\beta=15$ and $L=10$ domains defined by the boundaries $Y_{l}=\{2.8,3.6$, $\ldots, 9.2\}$, which gives the maximum value of the internal domain functions, given by Eq. (A7), of 0.9. If the population of the domain, $N_{l}=\sum_{k} w^{k} \Omega_{l}\left(y^{k}\right)$, is smaller than a threshold $\epsilon$ - the value used here is $\epsilon=0.01$-then this domain is added to the adjacent domain with higher population to form a wider domain of the functional form of Eq. (A4) according to Eq. (A5). These domain parameters, $\left\{Y_{l}, \beta, \epsilon\right\}$, were applied to other values of the initial collision momentum $P_{y}$. Between 5 and 25 thousand trajectories were propagated up to final time $T=3000$ a.u.

We examine the effect of the quantum force on the behavior of the vibrational DoF using several implementations of the LQF dynamics:

(i) The original LQF approach, ${ }^{23}$ where the approximation is determined on the entire space and depends on both, $x$ and $y$ coordinates. It is referred to as $\mathrm{LQF}^{2 \mathrm{D}}$.

(ii) The LQF is defined on the whole space assuming that it depends only on the quantum DoF. The corresponding results are referred to as $\mathrm{LQF}^{1 \mathrm{D}}$. This is the $L=1$ version of the $\mathrm{LQF}$ with classical domains. (iii) The LQF is defined on $L=10$ classical domains described above and terms containing domain derivatives are neglected, i.e., $\partial U / \partial y \approx 0$. The results are labeled $\mathrm{LQF}^{d o m}$.

(vi) The same quantum potential as in $\mathrm{LQF}^{\text {dom }}$ given by Eq. (16) with the domain derivatives included (the results are referred to as $\mathrm{LQF}^{\text {der }}$ ),

$$
\partial U / \partial y=\sum_{l} U_{l} \partial \Omega_{l} / \partial y .
$$

(v) The stabilized version of $\mathrm{LQF}^{\text {dom }}$ described in Subsection III B 2. It will be referred to as $\mathrm{LQF}^{\text {stb }}$.

Some average quantities for the wavepacket propagation using these different methods are listed in Table II and shown in Figs. 5 and 6. The average "post-collision" position $\langle x\rangle_{t}-$ the results are averaged over the last $25 \%$ of the propagation interval—is in good agreement with the QM result for all LQF methods. Let us examine the total energy plotted as functions of time in Fig. 5; note that for the $\mathrm{LQF}^{\text {dom }}$ calculation the energy deviation from the analytical value is scaled by a factor of 10: $E_{\text {plot }}=E(0)+(E(t)-E(0)) / 10$. Unlike $\mathrm{LQF}^{2 \mathrm{D}}$ and $\mathrm{LQF}^{1 \mathrm{D}}$ methods, the energy non-conservation in $\mathrm{LQF}^{d o m}$ calculation is appreciable; the largest deviation of $E$ from its initial value is $8 \%$ (see Table II for comparison). Inclusion of the $y$-derivatives of $U$ yields the energy-conserving dynamics, because the quantum force, now a vector, is given by the exact negative gradient of $U$. This energy, labeled $\mathrm{LQF}^{d e r}$ in

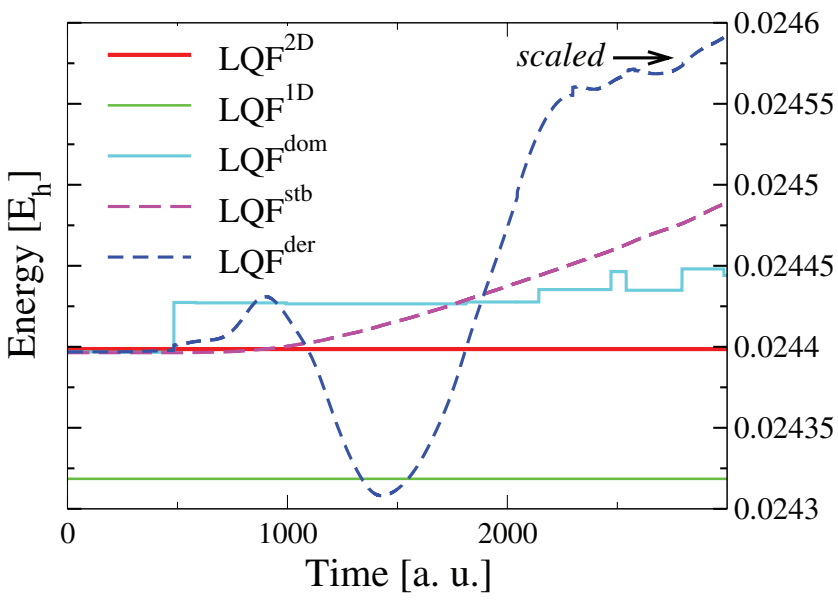

FIG. 5. Conservation of total energy for the $\mathrm{He}+\mathrm{HBr}$ model. On all panels the results are shown for the collision momentum $P_{Y}=-16 \sqrt{E_{h} m_{e}}$, and were obtained using LQF for both dimensions (red), using LQF only in $x$ coordinate (green), using LQF in $x$ with 10 domains in classical DoF with (cyan) and without the $y$-derivatives (blue), and with stabilization terms (magenta). 

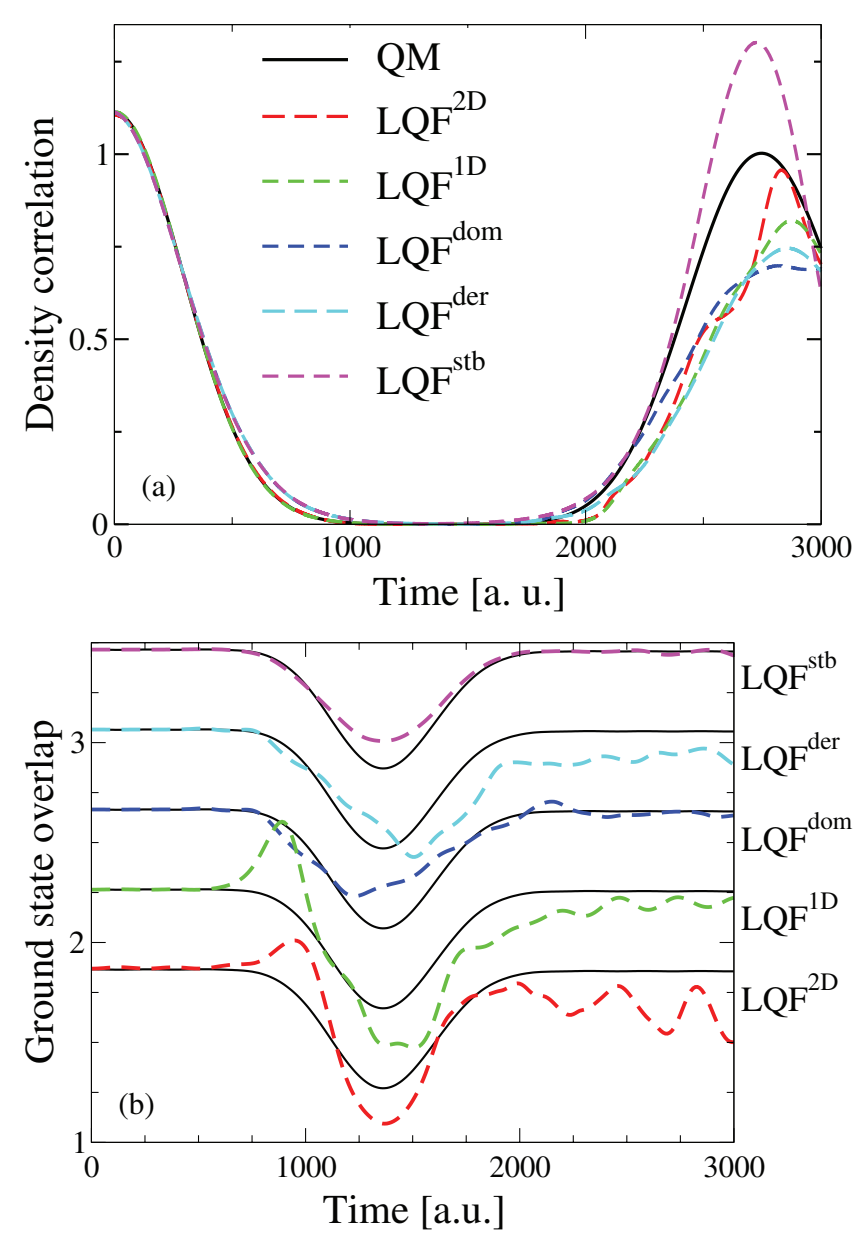

FIG. 6. $\mathrm{He}+\mathrm{HBr}$ model. On all panels the results are shown for the collision momentum $P_{0}=-16 \sqrt{E_{h} m_{e}}$ and obtained using LQF for both dimensions (red), using LQF only in $x$ coordinate (green), using LQF in quantum DoF with 10 domains in classical DoF with (cyan) and without the interface terms (blue), and with stabilization terms (magenta). In panel (b) the results obtained with the same as in (a) quantum trajectory methods are shifted along the y-axes by 0.4 units for different methods; the corresponding exact QM results are shown as solid lines. (a) Density overlap given by Eq. (36). (b) Overlap with the ground vibrational eigenstate given by Eq. (25).

Fig. 5, conserves energy within $0.2 \%$. This error comes from the jumps in $E(t)$ due to the merger of nearly empty domains (containing less than $\epsilon$ of the wavefunction density) with adjacent domains. Although the introduction of the quantum force into the classical DoF is formally correct, it deteriorates the description of the vibrational DoF as seen from the ground vibrational state overlaps in Fig. 6(b) and we view it as an artifact of the piecewise definition of the LQF on domains. Therefore, we turn to stabilization of dynamics without the built-in energy conservation.

\section{Stabilization of LQF dynamics on classical domains}

The modification of the LQF dynamics on classical domains, that will be referred to as $\mathrm{LQF}^{s t}$, is based on introduction of the stabilizing force, which cancels the leading term of the LQF-approximation error. The stabilization scheme, derived from the perturbation theory in Ref. 28 without the domains, is outlined first. Let us consider the classical and nonclassical components of the momentum operator, $\vec{p}$ of Eq. (4) and $\vec{r}$ of Eq. (13), on equal footing and within the same level of approximation. We will solve the evolution equations for $\vec{r}$ derivable from Eq. (9), which we did not considered after relating Eq. (9) to the constant trajectory weights of Eq. (11).

For the two-dimensional case in the Lagrangian frameof-reference the complete set of evolution equations is

$$
\begin{aligned}
\frac{d p_{x}}{d t} & =\left(\hat{\mathcal{K}}_{1}+\hat{\mathcal{K}}_{2}\right) r_{x}-\frac{\partial V}{\partial x}, \\
\frac{d p_{y}}{d t} & =\left(\hat{\mathcal{K}}_{1}+\hat{\mathcal{K}}_{2}\right) r_{y}-\frac{\partial V}{\partial y}, \\
-\frac{d r_{x}}{d t} & =\left(\hat{\mathcal{K}}_{1}+\hat{\mathcal{K}}_{2}\right) p_{x}, \\
-\frac{d r_{y}}{d t} & =\left(\hat{\mathcal{K}}_{1}+\hat{\mathcal{K}}_{2}\right) p_{y} .
\end{aligned}
$$

The differential operators on the RHS are

$$
\begin{gathered}
\hat{\mathcal{K}}_{1}=\frac{r_{x}}{m} \frac{\partial}{\partial x}+\frac{r_{y}}{M} \frac{\partial}{\partial y}, \\
\hat{\mathcal{K}}_{2}=\frac{1}{2 m} \frac{\partial^{2}}{\partial x^{2}}+\frac{1}{2 M} \frac{\partial^{2}}{\partial y^{2}} .
\end{gathered}
$$

We will define their action on $\vec{r}$ and $\vec{p}$ within the linear approximations to $\vec{r}=\left(r_{x}, r_{y}\right)$ and $\vec{p}=\left(p_{x}, p_{y}\right)$ (as done in LQF) resulting in approximations $\hat{\mathcal{K}}_{2} \vec{r} \approx 0$ and $\hat{\mathcal{K}}_{2} \vec{p} \approx 0$. Labeling the least square fits to the components of $\vec{r}$ and $\vec{p}$ with the tilde and adding the stabilization terms from Ref. 28 Eqs. (27) become

$$
\begin{aligned}
\frac{d p_{x}}{d t}+\frac{\partial V}{\partial x} & =\frac{r_{x}}{m} \frac{\partial \tilde{r}_{x}^{c}}{\partial x}+\frac{2}{m} \frac{\partial \tilde{r}_{x}}{\partial x}\left(r_{x}-\tilde{r}_{x}\right), \\
\frac{d p_{y}}{d t}+\frac{\partial V}{\partial y} & =\frac{r_{y}}{M} \frac{\partial \tilde{r}_{y}^{c}}{\partial y}+\frac{2}{M} \frac{\partial \tilde{r}_{y}}{\partial y}\left(r_{y}-\tilde{r}_{y}\right) \\
-\frac{d r_{x}}{d t} & =\frac{r_{x}}{m} \frac{\partial \tilde{p}_{x}^{c}}{\partial x}+\frac{2}{m} \frac{\partial \tilde{r}_{x}}{\partial x}\left(p_{x}-\tilde{p}_{x}\right) \\
-\frac{d r_{y}}{d t} & =\frac{r_{y}}{M} \frac{\partial \tilde{p}_{y}^{c}}{\partial y}+\frac{2}{M} \frac{\partial \tilde{r}_{y}}{\partial y}\left(p_{y}-\tilde{p}_{y}\right) .
\end{aligned}
$$

The last terms on the RHS of Eqs. (30) are the stabilization terms, which compensate for small errors in the quantum force due to linearization.

In Eqs. (30) the superscript $c$ denotes results of the coupled approximation to $\vec{r}$ and $\vec{p}$ introduced to conserve the total energy defined via $\vec{r}$, now computed for each trajectory:

$$
E^{r p}=\frac{\left\langle p_{x}^{2}+r_{x}^{2}\right\rangle}{2 m}+\frac{\left\langle p_{y}^{2}+r_{y}^{2}\right\rangle}{2 M}+\langle V\rangle .
$$

If Eqs. (27) are solved exactly, the sum of contributions $\left\langle r_{x}^{2}\right\rangle /(2 m)$ and $\left\langle r_{y}^{2}\right\rangle /(2 M)$ to the total energy is equivalent to the average value of the quantum potential of Eq. (7). The conservation of $E^{r p}$ is achieved when the following condition is fulfilled:

$$
I^{c}=\left\langle\vec{r} \cdot\left(\mathbf{F}^{r} \vec{p}-\mathbf{F}^{p} \vec{r}\right)\right\rangle=0,
$$

where $(a=\{x, y\}$ and $b=\{x, y\})$

$$
F_{a b}^{r}=\left\langle\partial \tilde{r}_{a}^{c} / \partial b\right\rangle, \quad F_{a b}^{p}=\left\langle\partial \tilde{p}_{a}^{c} / \partial b\right\rangle .
$$


Variables $a$ and $b$ stand for $x$ or $y$. Equation (32) is included into the least squares fit of $\vec{r}$ and of $\vec{p}$, minimizing

$$
I_{1}=\left\langle\left(r_{x}-\tilde{r}_{x}\right)^{2}\right\rangle \text { and } I_{2}=\left\langle\left(p_{x}-\tilde{p}_{y}\right)^{2}\right\rangle
$$

through the Lagrange multiplier. Thus, the coupled minimization of $I=I_{1}+I_{2}+2 \lambda I^{c}$ yielding $\tilde{r}^{c}$ and $\tilde{p}^{c}$, conserves the total energy of Eq. (31). Uncoupled minimization of $I_{1}$ and $I_{2}$ on the full space (no domains) yields $\tilde{r}$ and $\tilde{p}$ in the stabilization terms that still conserve the total energy.

We modify Eqs. (30) to include multiple classical domains dependent on $y, \Omega_{l}(y)$, as

$$
\frac{d p_{x}}{d t}+\frac{\partial V}{\partial x}=\frac{r_{x}}{m} \sum_{l=1}^{L} \frac{\partial\left(\tilde{r}_{x}^{c}\right)^{l}}{\partial x} \Omega_{l}(y)+\frac{2}{m} \sum_{l=1}^{L} \frac{\partial \tilde{r}_{x}^{l}}{\partial x}\left(r_{x}-\tilde{r}_{x}^{l}\right) \Omega_{l}(y),
$$

$-\frac{d r_{x}}{d t}=\frac{r_{x}}{m} \sum_{l=1}^{L} \frac{\partial\left(\tilde{p}_{x}^{c}\right)^{l}}{\partial x} \Omega_{l}(y)+\frac{2}{m} \sum_{l=1}^{L} \frac{\partial \tilde{r}_{x}^{l}}{\partial x}\left(p_{x}-\tilde{p}_{x}^{l}\right) \Omega_{l}(y)$.

The total energy is not formally conserved, because the fitting is performed for each domain independently (for practical reasons) and because the terms with derivatives of $\Omega_{l}$ are omitted (for the reasons stated earlier) but even so stabilization of dynamics significantly improves energy conservation compared to LQF on domains of Sec. II. The total energy computed with dynamics of Eqs. (35) shown in Fig. 5 deviates from its initial value by less than $0.4 \%$. The error grows with time, and it is desirable to develop in the future a stabilization scheme with bound energy error.

\section{Results and analysis for the vibrational degree of freedom}

Now let us focus on the quantities besides the total energy - average position of the quantum and classical DoFs, ground state overlap given by Eq. (25) and density correlation $C(t)$,

$$
C(t)=\iint|\psi(x, y, 0)|^{2}|\psi(x, y, t)|^{2} d x d y .
$$

An ensemble of 25000 trajectories was propagated using the five versions of LQF with and without the domains listed in Sec. III B 1 up to final time $T=3000$ a.u. Table II contains $\langle x\rangle_{t}$ and its deviation averaged over the post-collision time interval, $t=[0.75 T, T]$ a.u., $\langle y\rangle_{T}$ evaluated at the final time $T$, and the total energy of the system averaged over the entire propagation time $t=[0, T]$. As seen from the table, all methods reproduce $\langle x\rangle_{t}$ rather well: the difference with the $\mathrm{QM}$ result is $0.1 \%$ or better. The main difference between the methods is observed in the deviation from the average value, where the best agreement with QM result is achieved in the stabilized calculation. The error in $\langle y\rangle_{T}$ is larger than that of $\langle x\rangle_{t}$ reaching $6 \%$, but is smallest for the stabilized calculation as well $(1 \%)$. While these data suggest that the dynamics on domains with stabilization $\left(\mathrm{LQF}^{s t b}\right)$ is more accurate in this problem than the other implementations of LQF, one needs to examine more detailed quantities to make conclusions. After all, in the harmonic oscillator the average position is exact even if the quantum force is set to zero.

Figure 6(a) shows the time correlation of the wavefunction density with itself given by Eq. (36). For the globally approximated quantum force $\left(\mathrm{LQF}^{2 \mathrm{D}}\right.$ and $\mathrm{LQF}^{1 \mathrm{D}}, C(t)$ reaches its post-collision maximum at a later time compared to the QM result despite the fact the initial decay of $C(t)$ is reproduced very accurately in both cases. We can conclude that the effect of QM force in the classical DoF is indeed small, and that the global linear quantum force does not correctly reproduce the energy exchange between the trajectories during the collision. Stabilized dynamics with domains captures the position of the maximum of $C(t)$ correctly, though not its amplitude. Figure 6(b) shows the "least average" quantity, the ground state overlap $C_{0}(t)$. Both versions of the globally defined LQF exhibit a peak around $t=1000$ a.u., i.e., at the onset of the collision, and oscillations in $C_{0}(t)$ after the collision, absent in the QM calculation. These discrepancies are consistent with the results on Fig. 6(a)—global LQF does not reproduce correctly the collision dynamics. Dynamics with domains, with the domain-dependent $\mathrm{LQF}$ is a more flexible function, which gives more accurate description of the collision process. A formulation with built-in but arguably unphysical energy conservation gives lower level of agreement. The stabilized dynamics is the most accurate.

Figures 7-9 show the results of the stabilized LQF dynamics on the same 10 domains for a range of collision momenta, $P_{y}=\{-12,-16,-20,-24,-28,-32\} \sqrt{E_{h} m_{e}}$. For all $P_{y}$ values the density correlation reproduces the positions of the post-collision maxima and of the overlaps with the ground state density. For the latter the post-collision $C_{0}(t)$ exhibits oscillations of growing with increase in collision energy amplitude, but its time-average corresponds to the QM results. These oscillations follow the pattern of $\langle x\rangle$ shown in Fig. 9 as a function of time: the amplitude of oscillation for the post-collision $\langle x\rangle$ grows with the increase of the collision energy; this trend is reproduced, but somewhat exaggerated in the trajectory dynamics results. Since $C(t)$ has $\left(x-x_{e}\right)^{2}$ in the

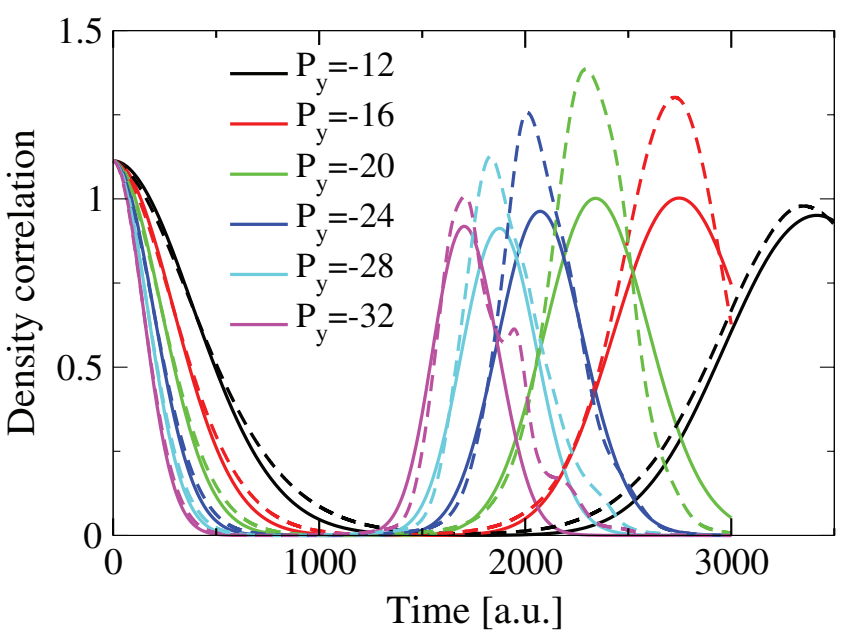

FIG. 7. $\mathrm{He}+\mathrm{HBr}$ model. The density correlation $C(t)$ given by Eq. (36) for the collision momentum $P_{0}=\{-12,-16,-20,-24,-28,-32\} \sqrt{E_{h} m_{e}}$. Stabilized LQF dynamics on domains (details are in text, the results are shown with dashes) is made to exact QM results (solid lines). 


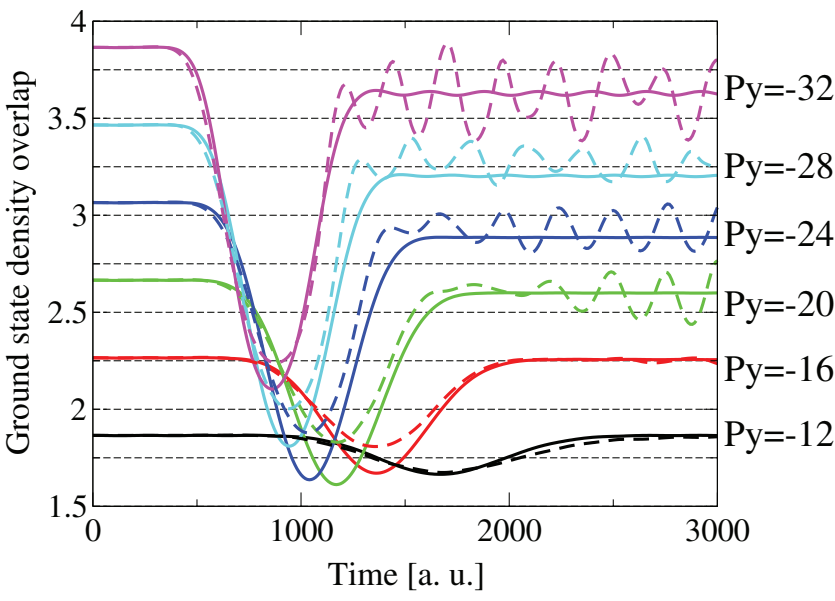

FIG. 8. He $+\mathrm{HBr}$ model. The ground state density overlap $C_{0}(t)$ given by Eq. (25) for the collision momentum $P_{0}=\{-12,-16,-20,-24,-28$, $-32\} \sqrt{E_{h} m_{e}}$. Stabilized LQF dynamics on domains (details are in text, the results are shown with dashes) is made to exact QM results (solid lines). The results for consecutive $P_{Y}$ are shifted along the vertical axis by 0.4 units.

exponent, the discrepancy with the QM result is exaggerated further, but in average captures the trend of increasing vibrational excitation with increase in $\left|P_{y}\right|$. The faster the collision the less time the displaced vibrational wavepacket has to return to the ground vibrational state showing smaller overlap with the ground vibrational state.

\section{CONCLUSIONS}

We have presented an approach of including QM effects on dynamics of nuclei into selected (so-called quantum) DoFs within the quantum trajectory formulation of TDSE for all DoFs. The quantum correction on dynamics is determined approximately using a linear fit to the nonclassical components of the momentum operator $\vec{r}=|\psi|^{-1} \nabla|\psi|$, generating LQF. Coordinates of only the quantum DoFs are included in the fit, but the dependence of the LQF parameters on the

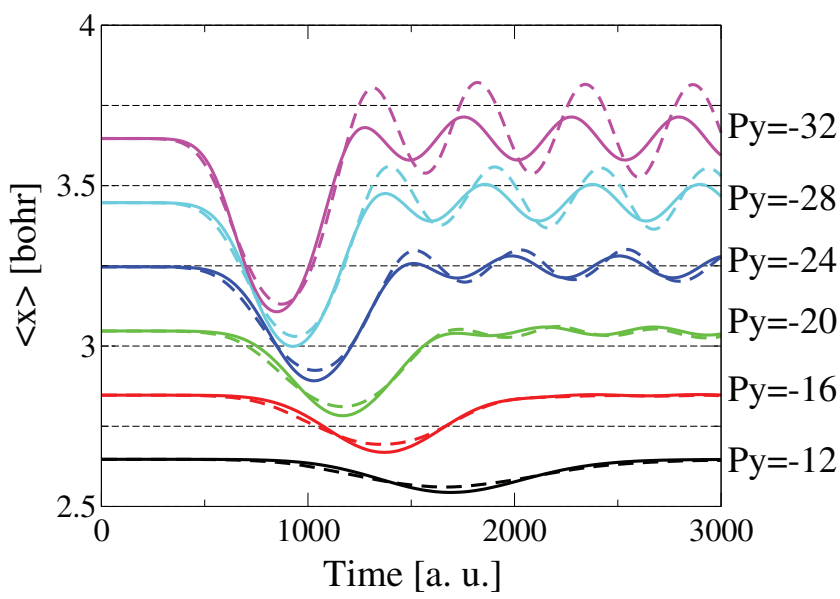

FIG. 9. He+HBr model. Average position for the quantum DoF, $\langle x\rangle$ for the collision momentum $P_{0}=\{-12,-16,-20,-24,-28,-32\} \sqrt{E_{h} m_{e}}$. Stabilized LQF dynamics on domains (details are in text, the results are shown with dashes) is made to exact QM results (solid lines). The results for consecutive $P_{Y}$ are shifted along the vertical axis by 0.2 units so-called classical DoFs is retained through spatial domains defined in those DoFs. The practical advantage of this strategy is a cheap, reduced dimensionality, quantum correction in the DoFs where QM effects are deemed important, e.g., into vibrational modes of light particles. Conceptually, multiple domains allow decoupling of the QM correction with respect to the classical DoFs: the QM correction is derived only from similar configurations of nearly classical particles.

Using several versions of the quantum trajectory dynamics with quantum corrections of reduced dimensionality we examined description of the zero-point-energy and vibrational excitations. We find that reduced dimensionality dynamics, when the objects such as wavefunctions or trajectories evolve in quantum and classical domains, generally affects the energy exchange between the modes of motion, as was the case for the $\mathrm{He}+\mathrm{HBr}$ model. Making a reduced dimensionality correction on dynamics performed in full space is a more general strategy. Out of several versions of the LQF dynamics, the dynamics with stabilization is the most promising as the most accurate in reproducing vibrational dynamics of $\mathrm{HBr}$ colliding with He. Further testing of the LQF on domains for chemically relevant model systems and finding a consistent way of choosing the classical domains are necessary before applying this method to studies of polyatomic systems. The convergence with respect to domain decomposition in the presented "minimalistic" two-dimensional models required large number of trajectories and was sensitive to the domain paremeters. We expect that in highdimensional systems, where hard quantum effects are generally quenched, the domain decomposition based on configurations of non-reactive DoFs will be appropriate. The mixed quantum/classical description of Refs. 13, 17, and 18, that may be interpreted as being related to the smallest domainseach defined by a single trajectory, is a limit of the domain decomposition. The methods were found useful in model and chemical systems, ${ }^{32-34}$ but more studies are certainly needed to develop a general domain decomposition scheme.

\section{ACKNOWLEDGMENTS}

Acknowledgment is made to the donors of the American Chemical Society Petroleum Research Fund for partial support of this research. Additional funding was provided by the Promising Investigator Research Award of the South Carolina Research Foundation and by the National Science Foundation under Grant No. CHE-1056188. The authors are grateful to V. A. Rassolov for many stimulating discussions.

\section{APPENDIX: CLASSICAL DOMAIN FUNCTIONS}

For a two-dimensional system, the domains in the classical DoF can be visualized as "strips" defined by the classical coordinate $y$. All trajectories $\left(x_{k}, y_{k}\right)$ for which $Y_{l-1}<y_{k}$ $<Y_{l}$ belong to the $l$ th domain. One can say that the quantum correction depends on $y$ parametrically through the domain index. The domain is defined by the Heaviside functions,

$$
\Omega_{l}(y)=\theta\left(y-Y_{l-1}\right)-\theta\left(y-Y_{l}\right), Y_{l}>Y_{l-1} .
$$



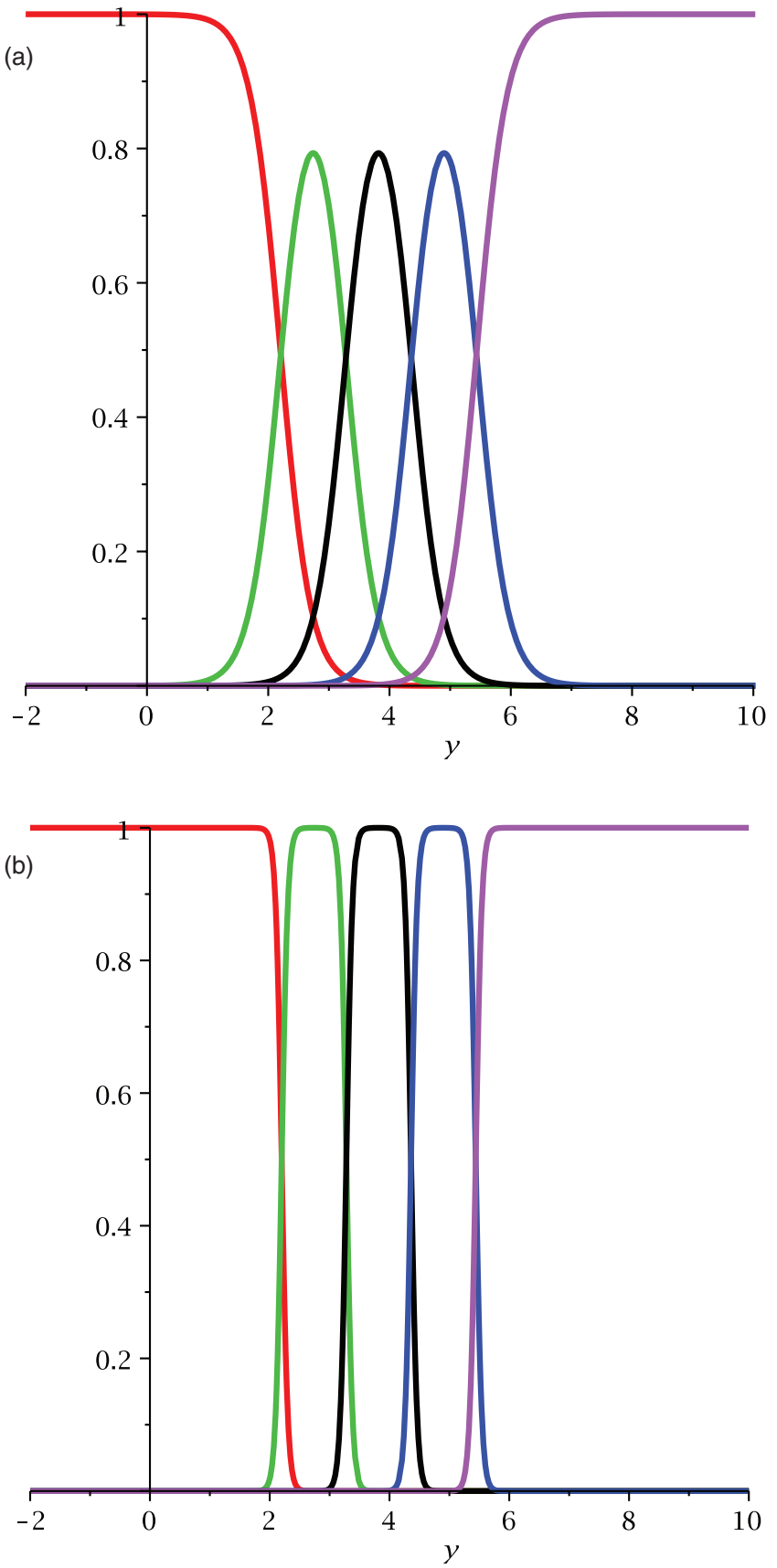

FIG. 10. Functions $\Omega_{l}$ defining $L=5$ domains with boundary spacing $Y_{l}-Y_{l-1}=1.08 a_{0}$ for $\beta=4 a_{0}^{-1}$ on the left panel and $\beta=20 a_{0}^{-1}$ on the right panel.

For numerical stability, the quantum force acting on a trajectory crossing the cell boundary has to be continuous. Therefore, instead of the Heaviside function we use its smooth version, the Fermi or logistic function in the limit of $\beta \rightarrow \infty$,

$$
f^{L}(y, Y)=\frac{1}{1+\exp (-\beta(y-Y))}-\frac{1}{2},
$$

for the left boundary and a complementary function for the right boundary,

$$
f^{R}(y, Y)=\frac{1}{1+\exp (\beta(y-Y))}-\frac{1}{2}=1-f^{L}(y, Y) .
$$

With these definitions, the adjacent domains are easy to add. Defining the domain function,

$$
\Omega\left(Y_{l-1}, Y_{l}\right)=f^{L}\left(y, Y_{l-1}\right)+f^{R}\left(y, Y_{l}\right),
$$

one obtains

$$
\Omega\left(Y_{l-1}, Y_{l}\right)+\Omega\left(Y_{l}, Y_{l+1}\right)=\Omega\left(Y_{l-1}, Y_{l+1}\right) .
$$

Choosing $Y_{0}=-\infty$ and $Y_{L}=\infty$, where $L$ is the number of domains,

$$
\sum_{l=1}^{L} \Omega\left(Y_{l-1}, Y_{l}\right)=\sum_{l=1}^{L} \Omega_{l}=1
$$

for any choice of the domain boundaries $Y_{l}$ or the slope parameter $\beta$. The maximum of the domain functions are

$$
\left.\Omega_{l}^{\max }\right|_{y=\left(Y_{l}-Y_{l-1}\right) / 2}=\tanh \left(\frac{\beta\left(Y_{l}-Y_{l-1}\right)}{4}\right) .
$$

Five domain functions are shown in Fig. 10. Domains with larger values of $\beta$ are more step-like but steeper slopes lead to more unstable trajectory dynamics.

${ }^{1}$ J. Kempe, A. Kitaev, and O. Regev, SIAM J. Comput. 35, 1070 (2006).

${ }^{2}$ V. A. Rassolov and S. Garashchuk, Chem. Phys. Lett. 464, 262 (2008).

${ }^{3}$ M. Karplus, R. D. Sharma, and R. N. Porter, J. Chem. Phys. 40, 2033 (1964).

${ }^{4}$ X. Zhu, P. Lopes, and A. D. MacKerell, Comput. Mol. Sci. 2, 167 (2012).

${ }^{5}$ C. Meier and U. Manthe, J. Chem. Phys. 115, 5477 (2001).

${ }^{6}$ G. Schiffel and U. Manthe, J. Chem. Phys. 132, 084103 (2010).

${ }^{7}$ W. H. Miller, J. Phys. Chem. A 105, 2942 (2001).

${ }^{8}$ G. C. Schatz, J. M. Bowman, and A. Kuppermann, J. Chem. Phys. 63, 685 (1975).

${ }^{9}$ R. Kosloff, J. Phys. Chem. 92, 2087 (1988).

${ }^{10}$ E. Madelung, Z. Phys. 40, 322 (1927).

${ }^{11} \mathrm{~L}$. de Broglie, An Introduction to the Study of Wave Mechanics (E. P. Dutton, New York, 1930).

${ }^{12}$ D. Bohm, Phys. Rev. 85, 166 (1952).

${ }^{13}$ O. V. Prezhdo and C. Brooksby, Phys. Rev. Lett. 86, 3215 (2001).

${ }^{14}$ W. H. Miller and C. W. McCurdy, J. Chem. Phys. 69, 5163 (1978).

${ }^{15}$ J. C. Tully, Faraday Discuss. 110, 407 (1998).

${ }^{16}$ J. C. Burant and J. C. Tully, J. Chem. Phys. 112, 6097 (2000).

${ }^{17}$ E. Gindensperger, C. Meier, and J. A. Beswick, J. Chem. Phys. 113, 9369 (2000).

${ }^{18}$ S. Garashchuk and M. V. Volkov, Mol. Phys. 110, 985 (2012).

${ }^{19}$ S. Habershon and D. E. Manolopoulos, J. Chem. Phys. 131, 244518 (2009).

${ }^{20}$ G. Czako and J. M. Bowman, J. Chem. Phys. 131, 244302 (2009).

${ }^{21}$ W. Zhang, H. Kawamata, and K. Liu, Science 325, 303 (2009).

${ }^{22}$ S. Garashchuk and V. A. Rassolov, Chem. Phys. Lett. 364, 562 (2002).

${ }^{23}$ S. Garashchuk and V. A. Rassolov, J. Chem. Phys. 120, 1181 (2004).

${ }^{24}$ S. Garashchuk and V. A. Rassolov, J. Chem. Phys. 118, 2482 (2003).

${ }^{25}$ S. Garashchuk and V. A. Rassolov, J. Phys. Chem. A 112, 10251 (2007).

${ }^{26}$ V. A. Rassolov and S. Garashchuk, J. Chem. Phys. 120, 6815 (2004).

${ }^{27}$ W. H. Press, B. P. Flannery, S. A. Teukolsky, and W. T. Vetterling, Numerical Recipes: The Art of Scientific Computing, 2nd ed. (Cambridge University Press, Cambridge, 1992).

${ }^{28}$ S. Garashchuk and V. A. Rassolov, J. Chem. Phys. 129, 024109 (2008).

${ }^{29}$ S. Garashchuk, J. Chem. Phys. 126, 154104 (2007).

${ }^{30}$ M. D. Feit, J. A. Fleck, Jr., and A. Steiger, J. Comput. Phys. 47, 412 (1982).

${ }^{31}$ C. Leforestier, R. H. Bisselling, C. Cerjan, M. D. Feit, R. Friesner, A. Guldberg, A. Hammerich, G. Jolicard, W. Karrlein, H. D. Meyer et al., J. Comput. Phys. 94, 59 (1991).

${ }^{32}$ E. Gindensperger, C. Meier, J. A. Beswick, and G. Parlant, J. Chem. Phys. 123, 214107 (2005).

${ }^{33}$ C. Meier, Phys. Rev. Lett. 93, 173003 (2004).

${ }^{34}$ E. Gindensperger, C. Meier, J. A. Beswick, and M. C. Heitz, J. Chem. Phys. 116, 10051 (2002). 